\title{
Revisiting the Role of Charge Transfer and Local Excitations in Thermally
}

\section{Activated Delayed Fluorescence}

Leonardo Evaristo de Sousa ${ }^{1}$ and Piotr de Silva ${ }^{1, \text { a) }}$

Department of Energy Conversion and Storage, Technical University of Denmark, Anker Engelunds Vej 301, 2800 Kongens Lyngby, Denmark

Thermally activated delayed fluorescence (TADF) is a phenomenon that relies on the upconversion of triplet excitons to singlet excitons by means of reverse intersystem crossing (rISC). It has been shown both experimentally and theoretically that the TADF mechanism depends on the interplay between charge transfer and local excitations. However, the difference between the diabatic and adiabatic character of the involved excited states is rarely discussed in the literature. Here, we develop a diabatization procedure to implement a 4-state model Hamiltonian to a set of TADF molecules. We provide physical interpretation for the Hamiltonian elements and show their dependence on the electronic state of the equilibrium geometry. We also demonstrate how vibrations affect TADF efficiency by modifying the diabatic decomposition of the molecule. Finally, we provide a simple model that connects the diabatic Hamiltonian to the electronic properties relevant to TADF and show how such relationship translates into different optimization strategies for rISC, fluorescence and overall TADF performance.

a)Electronic mail: pdes@dtu.dk 


\section{INTRODUCTION}

Recent research in organic light-emitting diodes (OLEDs) materials has increasingly focused on the phenomenon of thermally activated delayed fluorescence (TADF) as a means to improve device efficiency ${ }^{1-4}$. This is important because due to spin-statistics, only $25 \%$ of excitons generated upon electron-hole recombination in a device are singlet and thus emissive ${ }^{5}$. The remaining $75 \%$ are composed of triplet excitons that usually decay non-radiatively, constituting an efficiency loss mechanism. In this sense, the TADF mechanism comes as a possible solution for this efficiency limitation. It is based on a thermal up-conversion process in which triplets excitons are converted into singlets, allowing them to decay radiatively at a later time. Except for some rare cases ${ }^{6,7}$, the first excited triplet state $\left(T_{1}\right)$ is lower in energy than the first excited singlet state $\left(S_{1}\right)$. Therefore, this up-conversion process, dubbed reverse intersystem crossing (rISC), is limited by the capacity of the available thermal energy to overcome the energy barrier (the singlet-triplet gap). Furthermore, as the process involves a spin flip, it also depends on a non-zero spin-orbit coupling (SOC) between the $T_{1}$ and $S_{1}$ states. These requirements put some constraints on molecular design.

Early attempts at making TADF efficient enough for OLED applications focused on a molecular design that could minimize the singlet-triplet gap ${ }^{8-10}$. This has led to the ubiquitous donoracceptor architecture, in which electron donor and electron acceptor fragments are attached in such a way as to localize orbitals in either fragment, reducing their spatial overlap and, consequently, their exchange energy. This arrangement results in $S_{1}$ and $T_{1}$ states that have strong charge transfer (CT) character and whose energy gap is minimized, as it is given by twice the exchange energy. In this sense, the understanding of TADF was initially focused on this picture of a two-state model composed of two CT states.

An unwanted consequence of the strong CT character of the $S_{1}$ state is the low oscillator strength associated with the $S_{1} \rightarrow S_{0}$ transition $^{11}$. Such low oscillator values, in turn, translate into low fluorescence rates, partially denying the benefits of successful triplet exciton recycling. Better emissive properties, however, are characteristic of transitions from excited states with a local excitation (LE) character. More recently, the role of such local states has come forward as fundamental to the better understanding of the TADF phenomenon, indicating that the two-state model approach was insufficient to paint the whole picture ${ }^{12-17}$. This realization resulted from experimental ${ }^{18-20}$ works that pointed at the LE character of the $T_{1}$ state in some molecules and also from theoretical works that suggested the importance of vibronic coupling between CT and 
LE triplet states to explain observed rISC rates $^{21,22}$.

The CT and LE state labels are mostly used in literature to denote the dominant character of the adiabatic excited states. However, these states can generally have a mixed character with relative compositions that can be further modified by molecular vibrations. Therefore, a more rigorous way of discussing the CT/LE states is to treat them as diabatic representations ${ }^{23-26}$, i.e. states of a well-defined electronic character that does not depend on nuclear configuration. In contrast, the adiabatic states are those that diagonalize the electronic Hamiltonian, therefore have well-defined electronic energies. Since adiabatic states can be generally written as linear combinations of some diabatic states, the latter are useful to discuss the differences in the electronic character of various potential energy surfaces.

Following these observations, a theoretical framework for describing electronic states relevant for TADF was presented in the form of a 4-state model Hamiltonian that accounted for CT and LE diabatic states ${ }^{27}$. This model was able to provide new insights into the rISC mechanism, but still lacked a better physical interpretation of its elements as well as a proper connection to the electronic properties relevant to the mechanism. To fill this gap it is necessary to make the bridge between a model written in terms of diabatic (CT/LE) states and molecular properties that can be obtained by means of quantum chemical calculations that are, however, usually conducted in the adiabatic representation.

The translation process between adiabatic to diabatic representation can be performed by means of a diabatization procedure. Several diabatization methods are available and can be roughly divided into those that start by explicitly building diabatic states and those that resort to a transformation from adiabatic do diabatic representation. Among the former are methods based on constrained density functional theory ${ }^{28-31}$ or $\Delta \mathrm{SCF}^{32,33}$. The latter type includes projection methods in which the adiabatic to diabatic transformation is performed by projecting the adiabatic states onto a set of reference diabatic states ${ }^{34-36}$.

In this work, we make use of a projection-based diabatization procedure that is able to extract general $N$-state models from time-dependent density functional theory (TD-DFT) calculations performed in the adiabatic representation for several TADF molecules. We first focus our analysis on the molecules for which 4-state models are found suitable and we explore the connections between the elements of the model Hamiltonians and the molecules' electronic properties. In addition, we analyze the effects of excited versus ground state equilibrium geometries in the models and show its dependence on the diabatic decomposition of the electronic states. Furthermore, we consider 
vibration effects by applying the model to a vast sample of molecular conformations and show how vibrations act by modifying the diabatic character of the states relevant to TADF. Finally, we use the generated data to find the relationship between the elements of the model Hamiltonian and electronic properties such as the singlet-triplet gap and the oscillator strength. This allows us to uncover different optimization strategies for maximizing rISC, fluorescence and overall TADF performance.

\section{METHODS}

\section{A. The 4-state and N-state models of TADF}

In the simplest 2-state model of TADF, two adiabatic states $S_{1}$ and $T_{1}$ have a pure CT character and can be written as linear combinations of two spin-mixed configuration state functions $|C T 1\rangle$ and $|C T 2\rangle$ that differ by a spin flip of unpaired electrons. The 4-state $\operatorname{model}^{27}$, in contrast, comes up when one considers the possibility of adiabatic states being composed of a mixture between CT and LE diabatic states. In this case, one aims at writing the adiabatic states as a combination of two CT and two LE states with opposite spin. In this new $(|C T 1\rangle,|C T 2\rangle,|L E 1\rangle,|L E 2\rangle)$ basis, the Hamiltonian has the following form

$$
\mathbf{H}_{d}=\left(\begin{array}{cccc}
0 & K_{C T} & t & K_{X} \\
K_{C T} & 0 & K_{X} & t \\
t & K_{X} & \Delta E & K_{L E} \\
K_{X} & t & K_{L E} & \Delta E
\end{array}\right) .
$$

The energy of the CT states is subtracted from the diagonal, which means that $\Delta E$ is the energy difference between CT and LE states. The remaining terms are couplings between the different CT and LE states: $K_{C T}$ is the coupling between the two CT states, $K_{L E}$ couples the two LE states and $t$ is the coupling between CT and LE states.

The 4-state model is clearly more general than its 2-state counterpart, and can explain the role of LE states in TADF which enable appreciable rISC and fluorescence. However, it is by no means an ultimate diabatic Hamiltonian as more than single CT or LE configurations may be present in low-lying adiabatic states of TADF molecules. Therefore, one may further extend it by including any number $N$ of diabatic states, in which case we may see these two aforementioned models as particular cases of a more general $N$-state model. The appropriate number $N$ of states necessary 
to well describe a particular system could in principle vary from molecule to molecule, as these models rely on the assumption that the diabatic basis set is able to span the space corresponding to some set of $N$ adiabatic states. The challenge then becomes finding the minimal basis set necessary to analyze TADF for a given molecule. This is what the algorithm we present next aims at doing.

\section{B. Diabatization Scheme}

\section{Defining Diabatic States}

The diabatization procedure starts by running a calculation on the donor-acceptor molecule to obtain the energies of a fixed number of singlet and triplet excited states as well as a set of localized molecular orbitals. In our implementation we fixed the initial number of states to 10 and used Natural Localized Molecular Orbitals ${ }^{37}$ (NLMO). Once this calculation is done, the NLMOs are classified as belonging either to the donor fragment, to the acceptor fragment or to the bond between the two fragments. This is done by first writing a vector whose components are the NLMO coefficients in the atomic orbital basis $\left(\vec{C}_{N M L O}\right)$. This vector is then decomposed into three vectors, each corresponding to donor $\left(\vec{C}_{D}\right)$, acceptor $\left(\vec{C}_{A}\right)$ or bond $\left(\vec{C}_{B}\right)$ contributions. The decomposition is made by setting to zero all coefficients in the NMLO vector that do not correspond to atomic orbitals of the desired fragment. As such, we have

$$
\vec{C}_{N M L O}=\vec{C}_{D}+\vec{C}_{A}+\vec{C}_{B}
$$

The square of the inner product between $\vec{C}_{N M L O}$ and each individual component gives each fragment's contribution to the NLMO and the largest contribution is used to classify each orbital.

The next step involves the decomposition of the adiabatic states $\left(\Psi_{i}^{a}\right)$ in terms of the different diabatic components $\left(\Psi_{j}^{d}\right)$.

$$
\left|\Psi_{i}^{a}\right\rangle=\sum_{j=1}^{4} c_{i j}\left|\Psi_{j}^{d}\right\rangle
$$

The four diabats correspond to the following states: donor-to-acceptor charge transfer (CT), reverse (acceptor-to-donor) charge transfer (rCT), local excitations in the donor (LED) and local excitations in the acceptor (LEA). These diabats are defined as linear combinations of configuration state functions composed of all possible combinations of transitions between orbitals with given classifications. For example, the CT diabats are composed of all combinations of transitions 
from orbitals localized in the donor fragment to orbitals localized in the acceptor fragment. The coefficients of this decomposition are obtained by projecting the adiabatic state onto each of the diabats

$$
c_{i j}=\left\langle\Psi_{j}^{d} \mid \Psi_{i}^{a}\right\rangle
$$

Calculation of these coefficients allows us to quantify the contribution of each diabatic state to the overall norm of each adiabatic state. This will be necessary to select the appropriate diabatic states that are going to make up our $N$-state models.

In an $N$-state model, $N$ is necessarily an even number, as we include both spin configurations of every selected diabatic state. Furthermore, we require that among all diabatic states, at least one state with charge transfer character (either CT or rCT) and one with local character (either LED or LEA) be present. The goal then is to find the minimal set of $N$ diabatic states that is able

to reproduce at least a fraction $p^{2}$ of the squared norm of each of the corresponding $N$ adiabatic states $\left(N / 2\right.$ singlet and $N / 2$ triplet states). The higher the $p^{2}$ criterion, then typically the larger the number $N$ of states that must be included in the model. As such, this $p^{2}$ criterion serves as a measure of the model's quality, as it indicates the lower bound estimate of how well the selected diabatic states are able to describe their adiabatic counterparts.

\section{2. $\quad$ Selecting Model States}

The selection of the adiabatic and diabatic states that are going to compose the model is done in two parts, starting with a $p^{2}$ value of 0.9 . The first part goes as follows:

1. Sort the adiabatic states by alternating singlets and triplets starting from $S_{1}$ and $T_{1}$.

2. Following the order, decompose the adiabatic state into its diabatic components.

3. Select two adiabatic states: one for which the CT component amounts to at least $\left(1-p^{2}\right)$ of the total norm squared and one for which an LE component satisfies this requirement.

4. For the selected adiabatic state, sort its diabatic contributions to the norm from largest to smallest. Add to the model the minimum number of diabats (including both spin configurations) such that their contributions when combined with the contributions from any pre- 
viously selected diabats add up to at least $p^{2}$. If the previously selected diabats are already able to account for $p^{2}$ of the norm, do not add any other diabatic state.

Once the first two states are selected, the remaining states are chosen by following the following procedure:

1. Decompose all the adiabatic states that have not been selected into the diabats that have been included so far in the model.

2. Calculate the fraction of the norm that the available diabats are able to reproduce for each state.

3. Take the adiabatic state with the highest norm. If by adding this state, the number of singlet and triplet adiabatic states in the model differs by more than 1, skip it. Otherwise, select this state to the model.

4. For the selected adiabatic state, sort its diabatic contributions to the norm from largest to smallest. Add to the model the minimum number of diabats (including both spin configurations) such that their contributions when combined with the contributions from any previously selected diabats add up to at least $p^{2}$. If the previously selected diabats are already able to account for $p^{2}$ of the norm, do not add any other diabatic state.

5. Repeat the process for the remaining adiabatic states until the number of selected adiabatic states matches the number of selected diabats. If this cannot be achieved, reduce the $p^{2}$ by 0.1 and repeat the entire procedure.

\section{The Diabatic Hamiltonian}

With $N$ adiabatic and $N$ diabatic states selected, we can use the transformation matrix $\mathbf{C}$ whose components are the coefficients $c_{i j}$, to transform the adiabatic Hamiltonian $\left(\mathbf{H}_{a}\right)$ into the diabatic one $\left(\mathbf{H}_{d}\right)$.

To do so, we first orthogonalize the transformation matrix by doing ${ }^{36,38}$

$$
\mathbf{T}=\mathbf{C}\left(\mathbf{C}^{\dagger} \mathbf{C}\right)^{-1 / 2}
$$

Then, the diabatic Hamiltonian is given by 


$$
\mathbf{H}_{D}=\mathbf{T H}_{A} \mathbf{T}^{\dagger}
$$

\section{Computational Details}

Density functional theory (DFT) and its time-dependent counterpart (TD-DFT) were used to calculate all the electronic properties of interest. The long range corrected functional $\omega \mathrm{B} 97 \mathrm{XD}^{39}$ was used throughout along with the $6-31 \mathrm{G}(\mathrm{d}, \mathrm{p})$ basis set. The range parameter $(\omega)$ was tuned for each molecule following the procedure described in Ref. 39. The tuned functionals were then used to calculate optimized geometries at the $S_{0}, S_{1}$ and $T_{1}$ states. To calculate all the electronic properties relevant to the diabatization procedure, the same level of theory was used but with a fixed average value for the range parameter for all molecules $\left(0.154 \mathrm{bohr}^{-1}\right)$. This is done so as to facilitate the interpretation of the different results.

All calculations were run with the Gaussian $16^{40}$ software, except for spin-orbit couplings, which were calculated with QChem 5.2 ${ }^{41}$. Finally, the NLMOs were calculated with the NBO 3.1 package $^{42}$.

\section{Molecules}

A set of diverse donor-acceptor TADF molecules was chosen for application of the diabatization procedure. This set is composed of 1,3,6,8-tetramethylcarbazole-xanthone (MCZ-XT) ${ }^{43}, 4-(9 H-$ carbazol-9-yl)-3-(trifluoromethyl)benzonitrile (NCFCZ) ${ }^{44}$, phenothiazine-dibenzothiophene-S,Sdioxide (PTZ-DBTO2), ${ }^{45}$, phenothiazine-benzophenone (PTZ-BP) ${ }^{46}$, phenothiazine-xanthone $(\mathrm{PTZ}-\mathrm{XT})^{46}$, spiro[acridan-9,9'-Xanthene]-chromone (XAC-CM) ${ }^{47}$ and 10-phenyl-10H,10'Hspiro[acridine-9,9'-anthracen]-10'-one (ACRSA) ${ }^{48}$

\section{RESULTS}

Table I shows the $N$-state models selected by the algorithm when applied for various molecules using optimized geometries from the $S_{0}, S_{1}$ and $T_{1}$ states. These results correspond to the cases for which at least $80 \%$ of the norm of each of the selected adiabatic states is managed to be reproduced by the diabatic decomposition, which signals that the $N$-state model is appropriate. 


\begin{tabular}{|c|c|c|c|}
\hline Molecule & State Geometry & Number of States & Lowest Norm \\
\hline \multirow{3}{*}{ MCZ-XT } & $S_{0}$ & 4 & 0.863 \\
\hline & $S_{1}$ & 4 & 0.983 \\
\hline & $T_{1}$ & 4 & 0.983 \\
\hline \multirow{3}{*}{ NCFCZ } & $S_{0}$ & 4 & 0.872 \\
\hline & $S_{1}$ & 4 & 0.922 \\
\hline & $T_{1}$ & 6 & 0.961 \\
\hline \multirow{3}{*}{ PTZ-DBTO2 } & $S_{0}$ & 4 & 0.849 \\
\hline & $S_{1}$ & 8 & 0.882 \\
\hline & $T_{1}$ & 4 & 0.962 \\
\hline \multirow{3}{*}{ PTZ-BP } & $S_{0}$ & 4 & 0.953 \\
\hline & $S_{1}$ & 4 & 0.924 \\
\hline & $T_{1}$ & 6 & 0.934 \\
\hline \multirow{3}{*}{ PTZ-XT } & $S_{0}$ & 4 & 0.924 \\
\hline & $S_{1}$ & 4 & 0.948 \\
\hline & $T_{1}$ & 4 & 0.952 \\
\hline \multirow{3}{*}{$\mathrm{XAC}-\mathrm{CM}$} & $S_{0}$ & 4 & 0.884 \\
\hline & $S_{1}$ & 4 & 0.967 \\
\hline & $T_{1}$ & 4 & 0.975 \\
\hline \multirow{3}{*}{ ACRSA } & $S_{0}$ & 4 & 0.957 \\
\hline & $S_{1}$ & 4 & 0.981 \\
\hline & $T_{1}$ & 4 & 0.981 \\
\hline
\end{tabular}

TABLE I. $N$-state models selected by the diabatization algorithm for different TADF molecules at $S_{0}, S_{1}$ and $T_{1}$ state geometries. The lowest norm column shows the norm of the least well described adiabatic state used in the model and serves as a measure of quality of the selected model.

It is worth mention that these particular excited state geometries were selected because they are expected to be the most relevant geometries when considering ISC and rISC processes.

It can be seen in Table I that, in most cases, a 4-state model is able to be extracted from the adiabatic states. For MCZ-XT, PTZ-XT, XAC-CM and ACRSA the number of states in the diabatization procedure does not change when the electronic state of the optimized geometry is 

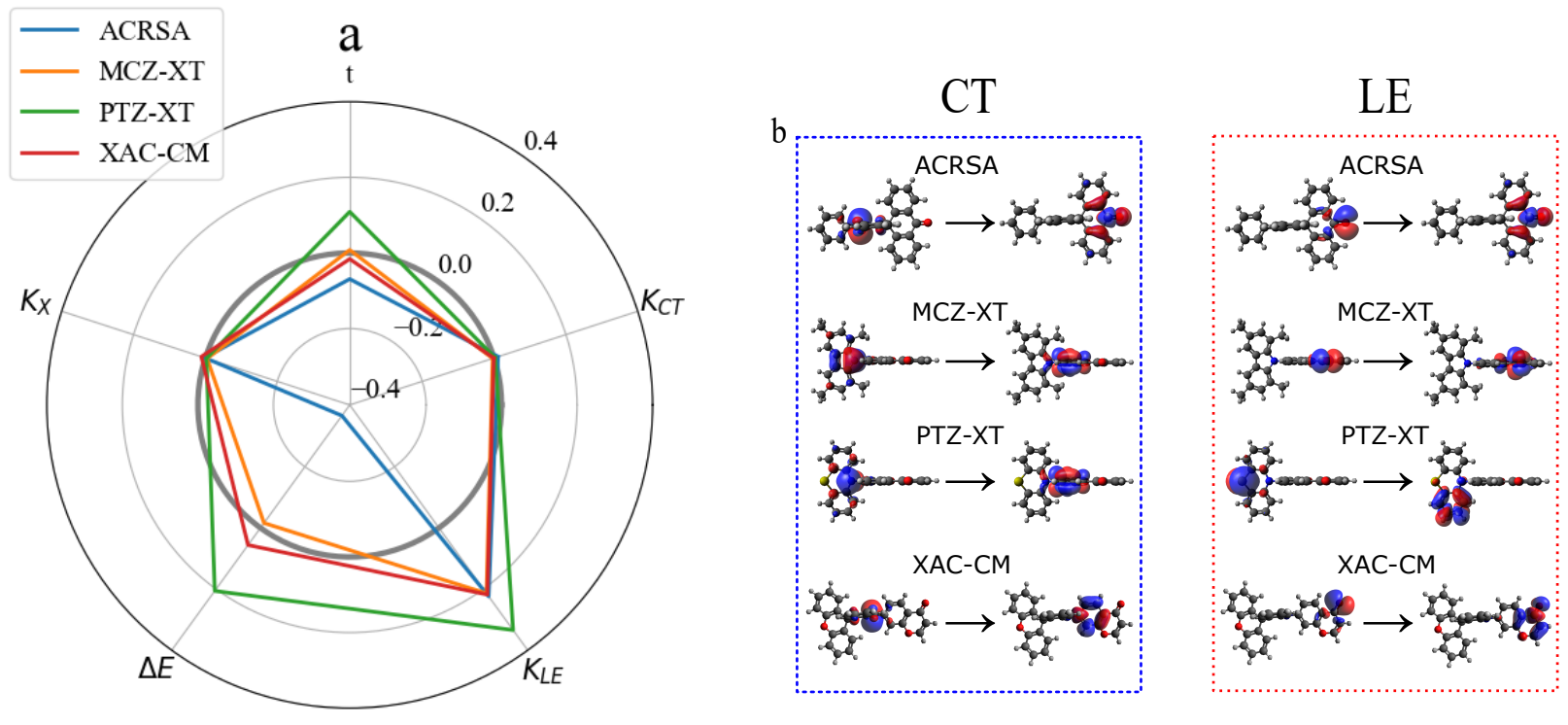

FIG. 1. a) Elements of the diabatic Hamiltonian (in $\mathrm{eV}$ ) obtained for four TADF molecules using their optimized ground state geometry. b) Orbital transitions that contribute the most to CT and LE diabatic state in the four TADF molecules.

modified from $S_{0}$ to $S_{1}$ or $T_{1}$. The same cannot be said of NCFCZ, PTZ-BP and PTZ-DBTO2. The first two molecules switch to a 6-state model when $T_{1}$ geometries are used, meaning that 4 states are not enough to reproduce at least 0.8 of the norms of the adiabats. For the $S_{1}$ geometry of PTZ-DBTO2, another 2 states are required, resulting in an 8-state model.

These examples show that changes in geometry can modify considerably the diabatization results. This can be mapped back to changes in the diabatic decomposition of the various adiabatic states and corroborates the importance of vibronic effects in the TADF mechanism. For example, the NCFCZ $T_{1}$ state is mostly a local excitation in the acceptor unit when calculated on $S_{0}$ and $T_{1}$ geometries. In the $S_{1}$ geometry it becomes a charge transfer state. A more dramatic example of such effect comes when considering the two equilibrium conformations of PTZ-DBTO2, the quasi-equatorial (whose results are shown in Table I) and quasi-axial conformations. Whereas for the quasi-equatorial conformation the algorithm manages to find suitable $N$-state models, for the quasi-axial conformer the lowest norm is at most 0.5 regardless of the geometry used, meaning that no subset of diabatic states was able to reproduce well the adiabatic states. This correlates with the fact that for the quasi-axial PTZ-DBTO2, the $S_{1}$ state does not possess a well defined electronic character, displaying rather a balanced mixture between CT and LEA diabats that are 
not necessarily present in other higher lying states. Interestingly, the change from quasi-equatorial to quasi-axial conformation is associated with a decreased TADF performance ${ }^{49}$.

With these issues in mind, we focus then on the molecules for which the algorithm produces suitable 4-state models for all geometries considered, namely ACRSA, MCZ-XT, PTX-XT and XAC-CM. Figure 1-a shows the five unique elements of the diabatic Hamiltonian obtained by applying the diabatization scheme to the $S_{0}$ geometries of the aforementioned molecules. The polar plot allows us to have a comprehensive view of how the diabatic Hamiltonian varies for each molecule. It stands out at first that two of the elements, the coupling between CT states $\left(K_{C T}\right)$ and the coupling between CT and LE states of opposite spin configuration $\left(K_{x}\right)$, are close to zero for all molecules. This is justified by the fact that these couplings rely on the existence of overlap between orbitals localized in distinct parts of the molecule. This can be visualized in Figure 1-b, which shows the orbital transition that contributes the most to each diabatic state. As both $K_{x}$ and $K_{C T}$ depend on overlaps between orbitals localized in the donor and the acceptor, these couplings end up close to zero when compared to the remaining terms.

The other two coupling terms, $K_{L E}$ and $t$, can have nonzero values. The former is the coupling between local states, and it is seen to amount to around $0.2 \mathrm{eV}$ for all but the PTZ-XT molecule, for which the coupling is $0.334 \mathrm{eV}$. It is worth mention that PTZ-XT is also the only molecule among the four for which the local state is located in the donor fragment, as it can be seen in Figure 1-b. The last coupling, $t$, is given by the sum of a hopping integral between the LE and CT states of same spin configuration and a two electron term that is typically close to zero as it also involves overlaps between orbitals localized at different molecular fragments. As such, the first term dominates, and $t$ can be interpreted as the coupling for electron transfer between donor and acceptor.

Figure 1-a shows that for MCZ-XT and XAC-CM the $t$ coupling is around zero, but the other two molecules, ACRSA and PTZ-XT, have non-zero $t$ values. This can be explained in terms of the diabatic decomposition of the adiabatic states that make up their 4-state models. In Figure 2 this decomposition can be seen. It stands out that for the two molecules for which $t$ is close to zero, the lowest energy states that have opposite diabatic character $\left(S_{1}\right.$ and $T_{1}$, in the case of MCZ-XT and XAC-CM) present practically no mixture of diabatic components, rather being almost pure CT and LE states. The opposite can be said of ACRSA and PTZ-XT. For these two, the lowest energy CT and LE states ( $S_{1}$ and $S_{2}$ for ACRSA; $S_{1}$ and $T_{1}$ for PTZ-XT) present significant admixture of the opposite diabatic component. As such, mixed CT/LE states are associated with higher absolute 

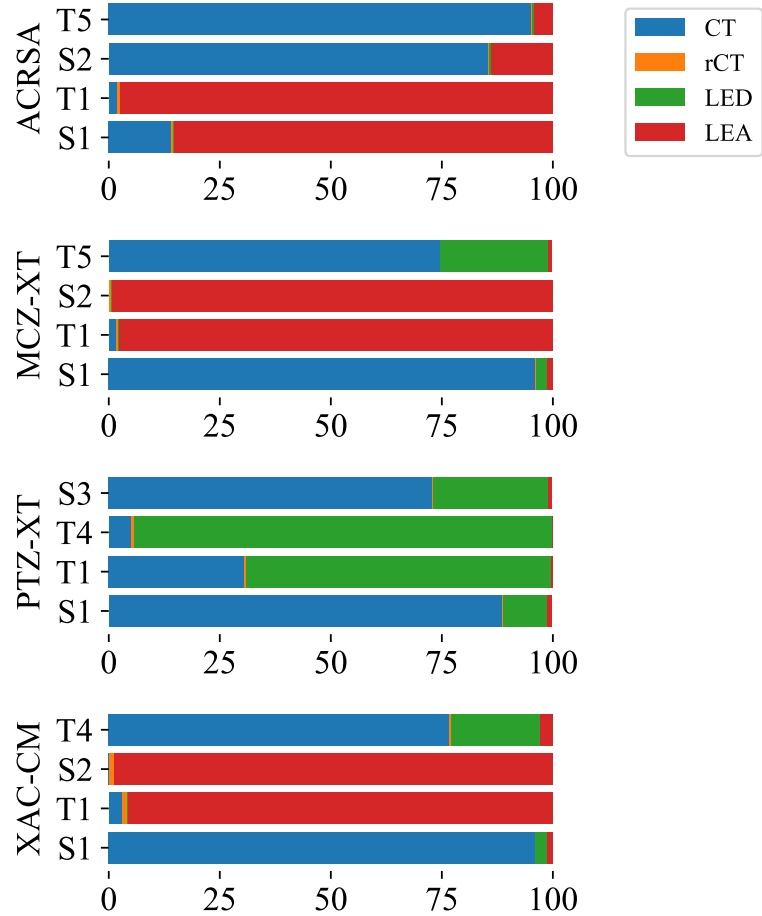

FIG. 2. Diabatic decomposition of each of the states that make up the 4-state models for ACRSA, MCZ-XT, PTZ-XT and XAC-CM under $S_{0}$ optimized geometries.

values of the $t$ coupling. It is worth noting that the sign of the coupling should not be important, as its contribution to transfer rates, for instance, depends on its square. Finally, the remaining term of the diabatic Hamiltonian, $\Delta E$, displays greater variability, going as high as $0.206 \mathrm{eV}$ for PTZ-XT and as low as $-0.367 \mathrm{eV}$ for ACRSA, as seen in Figure 1-a. Negative $\Delta E$ values are sure to be obtained when the higher energetic singlet and triplet states of the model have prevalent CT character. This is the case of ACRSA, in which $S_{2}$ and $T_{5}$ are the states with largest CT character. Conversely, to ensure positive $\Delta E$ values would require that the higher energy singlet and triplet states be of LE character. If this alignment does not take place either way, then it is possible to find both positive and negative $\Delta E$ values, as well as values closer to zero, as is the case for both MCZ-XT and XAC-CM. This will depend on the energies of the particular adiabatic states involved.

With a better understanding of the physical meaning of the elements of the diabatic Hamiltonian, we turn to question of how molecular geometries may affect the diabatization procedure. We start by comparing the diabatic Hamiltonians computed at $S_{0}, S_{1}$ and $T_{1}$ optimized geometries of the four molecules analyzed so far. These two excited states are expected to be even more relevant 
for the study of TADF molecules, as these are the states that mostly take part in intersystem crossing. This comparison is shown in Figure 3. First we note that $K_{X}$ and $K_{C T}$ couplings remain close to zero regardless of geometry for the reasons explained above. A second point that stands out is the fact that the $K_{L E}$ coupling appears to be independent of geometry, which indicates that the LE states used in the diabatization are very similar. This results from the fact that the individual donor/acceptor fragments in these molecules are rather rigid, and geometry optimization preserves their shape, even if its relative orientation with respect to the other fragment may have changed. As such, the local excited states experience little change from one optimized geometry to the other. In this sense, it is expected to find greater variability in the components of the diabatic Hamiltonian that depend on CT states. This is indeed the case of $t$ and especially $\Delta E$.

Concerning the $t$ coupling, we mentioned that it approaches zero when the adiabatic states present little mixture between diabatic components. This is clearly seen for both ACRSA and PTZ-XT. In the case of the former, only in the $S_{0}$ geometry we observe some mixture between CT and LE states (Figure 2), whereas in the other geometries we see two pure CT and two pure LE states. This can be seen in the decomposition diagrams for the 4-state models in the $S_{1}$ and $T_{1}$ geometries that are present in the Supporting Information file (Figures SI-1-a and SI-1-b). For PTZ-XT, $t$ has its highest absolute values for the $S_{0}$ geometry, which is the case where we observe the most mixture of diabatic components among all three geometries. The remaining molecules show no significant change neither in the values of $t$ nor in the degree of mixture of diabatic states in the composition of the adiabatic states the make up their 4-state model.

As to $\Delta E$, we note two distinct behaviors. For ACRSA and MCZ-XT, $\Delta E$ decreases to similar values when the 4-state model is obtained from the $S_{1}$ and $T_{1}$ geometries in comparison to the result from the $S_{0}$ geometry. This is explained by the fact that in these geometries the higher energy singlet and triplet state of the 4-state model have predominant CT character, whereas $S_{1}$ and $T_{1}$ have LEA character (see Figures SI-1-a and SI-1-b). On the other hand, PTZ-XT and XAC-CM display increased $\Delta E$ values when comparing results from $S_{0}$ and $S_{1}$ geometries and lower values for $T_{1}$ geometries. In both cases we have an increase in CT character of the $S_{1}$ and $T_{1}$ states at the $S_{1}$ geometry and, conversely, an increase in LE character of these same states for the $T_{1}$ geometry.

The above results show that the electronic state of a molecule may affect significantly its diabatic Hamiltonian, which, in turn, may change the electronic properties relevant for TADF. The relationship between electronic properties and the Hamiltonian requires further investigation. One 

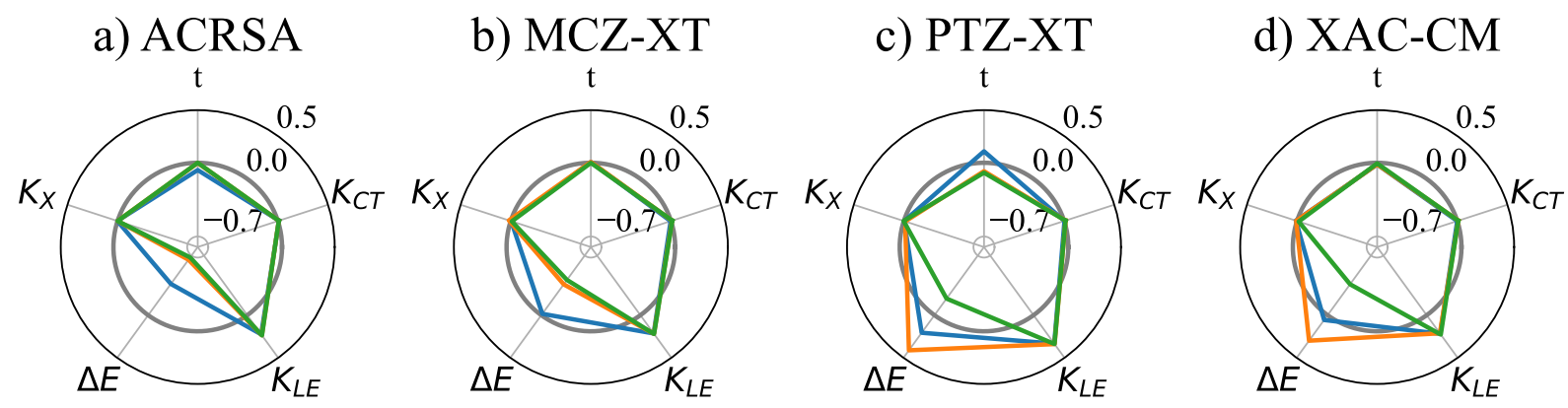

FIG. 3. Comparison between diabatic Hamiltonians calculated using the optimized geometries at the $S_{0}, S_{1}$ and $T_{1}$ states. All values in $\mathrm{eV}$.

such example of electronic property is the singlet-triplet gap $\left(\Delta E_{S T}\right)$, which can be calculated from the diabatic Hamiltonian by means of the following expression ${ }^{27}$ :

$$
\begin{aligned}
& \Delta E_{S T}=K_{L E}+K_{C T} \\
& \pm \sqrt{1 / 4\left(\Delta E+K_{L E}+K_{C T}\right)^{2}-\left(\Delta E+K_{L E}\right) K_{C T}+\left(t+K_{X}\right)^{2}} \\
& \pm \sqrt{1 / 4\left(\Delta E-K_{L E}-K_{C T}\right)^{2}-\left(\Delta E-K_{L E}\right) K_{C T}+\left(t-K_{X}\right)^{2}}
\end{aligned}
$$

If we account for our observations that $K_{X}$ and $K_{C T}$ should be close to zero and neglect these terms, we obtain a simplified version of the expression:

$$
\Delta E_{S T}=K_{L E} \pm \sqrt{1 / 4\left(\Delta E+K_{L E}\right)^{2}+t^{2}} \pm \sqrt{1 / 4\left(\Delta E-K_{L E}\right)^{2}+t^{2}}
$$

An interesting result follows for the case in which the coupling $t$ (or rather $t^{2}$ ) can also be neglected. As mentioned above, this would be the case for systems in which the adiabatic states that make up the 4-state model correspond to practically pure CT or LE states. If we account for 
the four possible sign configurations, we get four possible values for the singlet-triplet gap

$$
\Delta E_{S T}=\left\{\begin{array}{c}
0 \\
K_{L E}-\Delta E \\
K_{L E}+\Delta E \\
2 K_{L E}
\end{array}\right.
$$

These possibilities correspond to the four possible pure diabatic configurations of the $S_{1} / T_{1}$ states, namely, CT/CT, CT/LE, LE/CT and LE/LE. When both states are of CT character, the energy difference between them is given by $2 K_{C T}$, which tends to zero since $K_{C T}$ is negligible. Conversely, when both states are local excitations, the energy gap is given by $2 K_{L E}$. The two remaining cases are the mixed CT/LE and LE/CT configurations, which result in energy gaps given by $K_{L E}-\Delta E$ and $K_{L E}+\Delta E$, respectively. These observations are confirmed by calculating these different possible gap values and comparing them to the actual $S_{1}-T_{1}$ gaps obtained directly from DFT calculations. These results are shown in Table III, where it can be seen that in the cases in which $t$ is negligible, the proper singlet-triplet gap is reproduced by one of the four possibilities in Equation 9 according to their $S_{1} / T_{1}$ diabatic configuration.

After having analyzed the behavior of the diabatic Hamiltonian under different equilibrium geometries, we turn to the issue of vibrations. Vibronic effects have been proposed to affect considerably the ISC rates in TADF molecules ${ }^{21}$. As such, it is important to take such effects into account. Recently, we have proposed an approach to include vibrational effects in the calculations of ISC rates by means of sampling molecular geometries from a distribution that accounts for the molecule's normal modes and temperature ${ }^{50}$. In line with this idea, we have sampled 1000 geometries using as starting point the optimized geometry at the $S_{1}$ state of the NCFCZ molecule. As it can be seen in Table I, for this electronic state the NCFCZ molecule can be well represented by a 4-state model. The temperature for the distribution was set at $500 \mathrm{~K}$ so that we could explore a larger portion of the configuration space. Next, the diabatization procedure was applied to all conformations, but the algorithm restricted the model size to 4-state models only. This is done so that we can make comparisons across all conformations. Importantly, the overall quality of the generated models is high, as measured by the portion of each state's norm that is able to be reproduced by the model (Figure SI-2).

Figure 4-a shows the elements of the diabatic Hamiltonian for each of the sampled geometries (in blue) along with the results for the optimized geometry at $S_{1}$ (in red). We first observe that 


\begin{tabular}{cccccccc}
\hline Molecule & Geometry & $t$ & $\Delta E_{S T}$ & $S_{1} / T_{1}$ & $K_{L E}-\Delta E$ & $K_{L E}+\Delta E$ & $2 K_{L E}$ \\
\hline \multirow{3}{*}{ ACRSA } & $S_{0}$ & -0.069 & 0.432 & LE/LE & 0.591 & -0.143 & 0.448 \\
& $S_{1}$ & -0.002 & 0.471 & LE/LE & 0.886 & -0.416 & 0.470 \\
& $T_{1}$ & 0.000 & 0.475 & LE/LE & 0.915 & -0.441 & 0.474 \\
\hline \multirow{2}{*}{ MCZ-XT } & $S_{0}$ & 0.008 & 0.227 & CT/LE & 0.230 & 0.198 & 0.428 \\
& $S_{1}$ & 0.008 & 0.433 & LE/LE & 0.580 & -0.144 & 0.436 \\
& $T_{1}$ & -0.001 & 0.439 & LE/LE & 0.634 & -0.194 & 0.440 \\
\hline \multirow{3}{*}{ PTZ-XT } & $S_{0}$ & 0.109 & 0.178 & CT/LE & 0.128 & 0.540 & 0.668 \\
& $S_{1}$ & -0.080 & 0.053 & CT/CT & -0.073 & 0.751 & 0.678 \\
& $T_{1}$ & -0.094 & 0.516 & CT/LE & 0.533 & 0.143 & 0.676 \\
\hline \multirow{2}{*}{ XAC-CM } & $S_{0}$ & -0.016 & 0.165 & CT/LE & 0.162 & 0.274 & 0.436 \\
& $S_{1}$ & -0.015 & 0.025 & CT/CT & -0.087 & 0.511 & 0.424 \\
& $T_{1}$ & -0.006 & 0.451 & LE/LE & 0.590 & -0.136 & 0.454 \\
\hline
\end{tabular}

TABLE II. Comparing DFT calculated singlet-triplet gaps $\left(\Delta E_{S T}\right)$ with three possible gaps taken from Equation III for 4-state models calculated with 3 different geometries for 4 molecules. The prevalent diabatic character of the $S_{1}$ and $T_{1}$ states in each case are also shown. Values for the $t$ couplings are presented so that the appropriateness of the $t=0$ approximation may be evaluated. All numerical values are in units of eV.

the Hamiltonian of the optimized geometry presents near zero $t$ coupling and high $\Delta E$. Following the discussion above, we may infer two things from these observations: first, that the adiabatic states composing the model have almost pure diabatic character, meaning no mixture of diabatic states. Second, since $\Delta E$ is high, then the lower energy states $\left(S_{1}\right.$ and $\left.T_{1}\right)$ are more likely to be of CT character. Comparing the results of the optimized geometry with those from the various sampled configurations (see Figure SI-3 for the distributions of parameters) we note that these non-equilibrium geometries produce significant deviations in the $t$ and $K_{L E}$ couplings as well as in $\Delta E$. The $t$ couplings moving away from zero indicates that the adiabatic states present some degree of mixture of CT and LE characters, which, in turn, affects the $\Delta E$ values. These observations are confirmed by comparing the diabatic decomposition of $S_{1}$ and $T_{1}$ states in the optimized geometry versus the mean decomposition that accounts for all sampled conformations. These are shown in Figure 4-b. As expected, the states in the optimized geometry (Opt T1 and Opt S1) have almost 

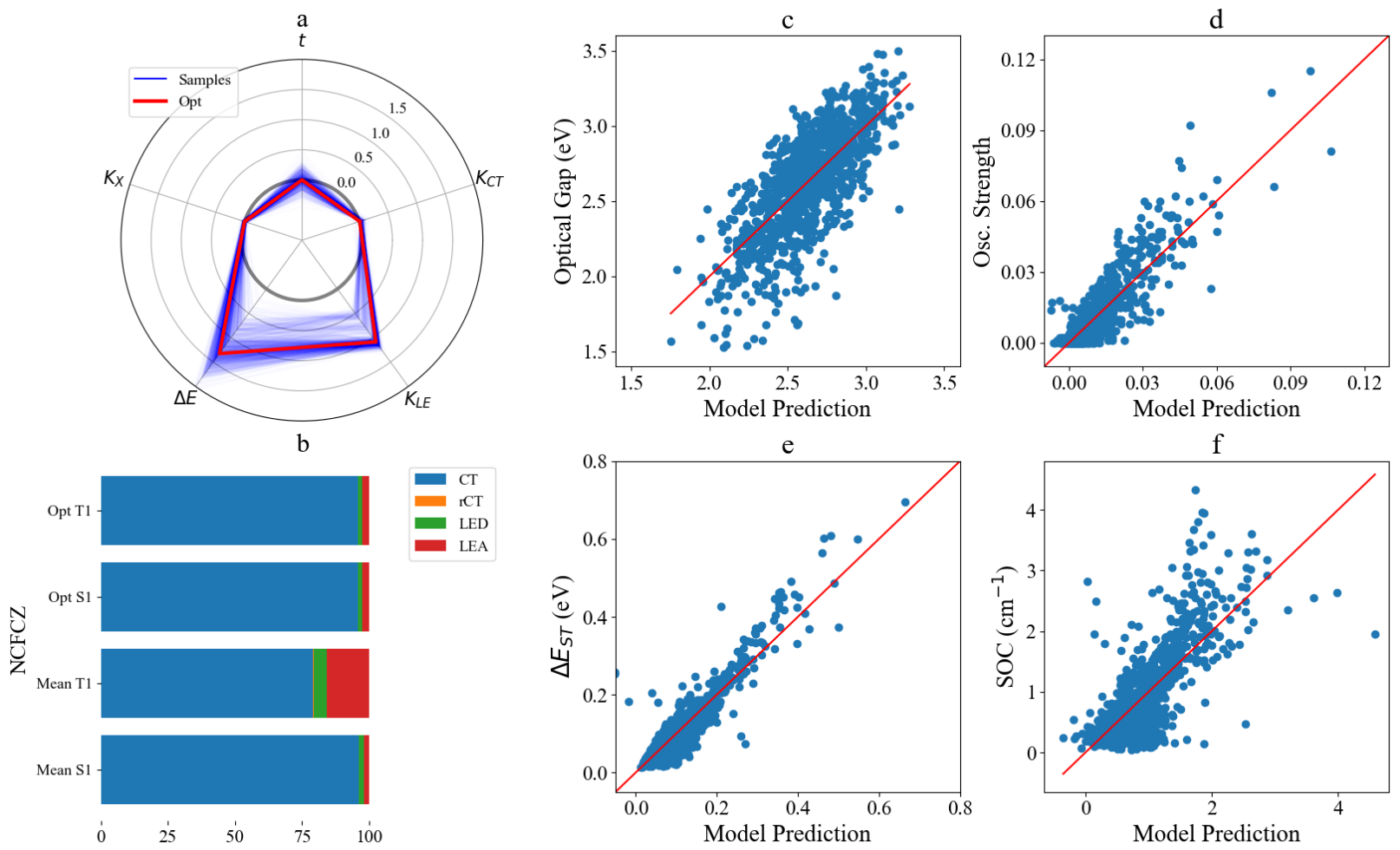

FIG. 4. a) Elements of the diabatic Hamiltonian (in eV) for the NCFCZ molecule in its $S_{1}$ state optimized geometry (red) and for 1000 sampled conformations produced by molecular vibrations (blue). b) Diabatic decomposition of the $S_{1}$ and $T_{1}$ states in the optimized $S_{1}$ geometry (Opt S1 and Opt T1) and mean diabatic decomposition of the 1000 sampled conformations (Mean S1, Mean T1). c) Calculated vs predicted values for optical gap, d) oscillator strength, e) singlet-triplet gap and f) spin-orbit coupling. The red lines mark the diagonal.

pure CT character. In addition, the average diabatic configuration of the ensemble of geometries does not change very much for the $S_{1}$ state (Mean $\mathrm{S} 1$ ). This is not the case, however, for the $T_{1}$ state, whose average decomposition gains a 15\% contribution from LEA states. It also displays an almost 5\% contribution of LED states. This means that for some configurations, the local diabatic state shifts from the acceptor to the donor. This is responsible for the region with low color density that presents both lower $K_{L E}$ and lower $\Delta E$. As these two parameters play a key role in the determination of the singlet-triplet gap, this result shows that vibrational effects may affect ISC rates in part by altering the singlet-triplet gap by means of changes in the diabatic decomposition of its $S_{1}$ and $T_{1}$ states. This observation prompts the question of what kind of changes could be expected in other relevant features. 
In devices whose operation rely on TADF, two properties are paramount: the rates of reverse ISC and fluorescence. These, in turn, depend mainly on two electronic properties each. For fluorescence, the relevant properties are the optical gap $\left(\Delta E_{o p t}\right)$ and the oscillator strength $(f)$ of the $S_{1} \rightarrow S_{0}$ transition. For rISC, these are the singlet-triplet gap $\left(\Delta E_{S T}\right)$ and the spin-orbit coupling (SOC) between $S_{1}$ and $T_{1}$ states. In this sense, we calculated these properties for all the sampled conformations of the NCFCZ molecule. To analyze the results, we applied a simple LASSO linear regression model ${ }^{51,52}$ with 5 -fold cross validation using as model features the elements of the diabatic Hamiltonian and all possible quadratic combinations thereof (regression coefficients are presented in Table SI-II). The comparison between model results and calculated values can be seen in Figures 4-c to $\mathrm{f}$. This simple model manages to capture the correct tendency for all features, but shows better predictive power for oscillator strengths $\left(R^{2}=0.79\right)$ and $\Delta E_{S T}\left(R^{2}=0.84\right)$, than for the optical gap $\left(R^{2}=0.55\right)$ and SOC $\left(R^{2}=0.57\right)$. In possession of a working relationship between the diabatic Hamiltonian and the electronic properties, it is worth investigating how the diabatic Hamiltonian looks like when seeking to optimize TADF performance.

Maximizing TADF performance entails optimizing fluorescence $\left(k_{f}\right)$ and rISC $\left(k_{r I S C}\right)$ rates simultaneously. As such, we look for the diabatic Hamiltonians that are able to maximize the product between $k_{f}$ and $k_{r I S C}$. We also look for diabatic Hamiltonians that are able to maximize each of the rates individually, so we may understand the trade-offs involved and we also attempt optimization of the individual underlying features. To do so, we employ a genetic algorithm coupled to the models shown in Figure 4-c to f. A detailed account of this procedure is provided in the SI file.

Figure 5-a shows diabatic Hamiltonians that maximize the three criteria (rISC, fluorescence and overall TADF). When the optimization procedure is applied to $k_{f}$ (green lines), it pushes $K_{C T}$ values higher indefinitely. The same behavior is observed when we attempt to maximize the oscillator strength (see Figure SI-4), which allows us to conclude that $K_{C T}$ is a major driver of $f$. Increasing $K_{C T}$, however, amounts to minimizing the spatial separation of CT orbitals. As such, the maximization of $K_{C T}$ effectively means that the state in question is rather turning into a LE state. In addition, the optimized Hamiltonian presents negative $\Delta E(-0.106 \mathrm{eV})$, which means that LE states should be the lower energy ones. Such behavior is expected, as CT states are known to display low transition dipole moments, so for efficient emission it is better that $S_{1}$ states have LE character. The optimized Hamiltonian also shows an elevated $t$ coupling $(0.08 \mathrm{eV})$, which is associated with increased admixture of LE and CT states in the $S_{1}$ state. We associate larger $t$ with 

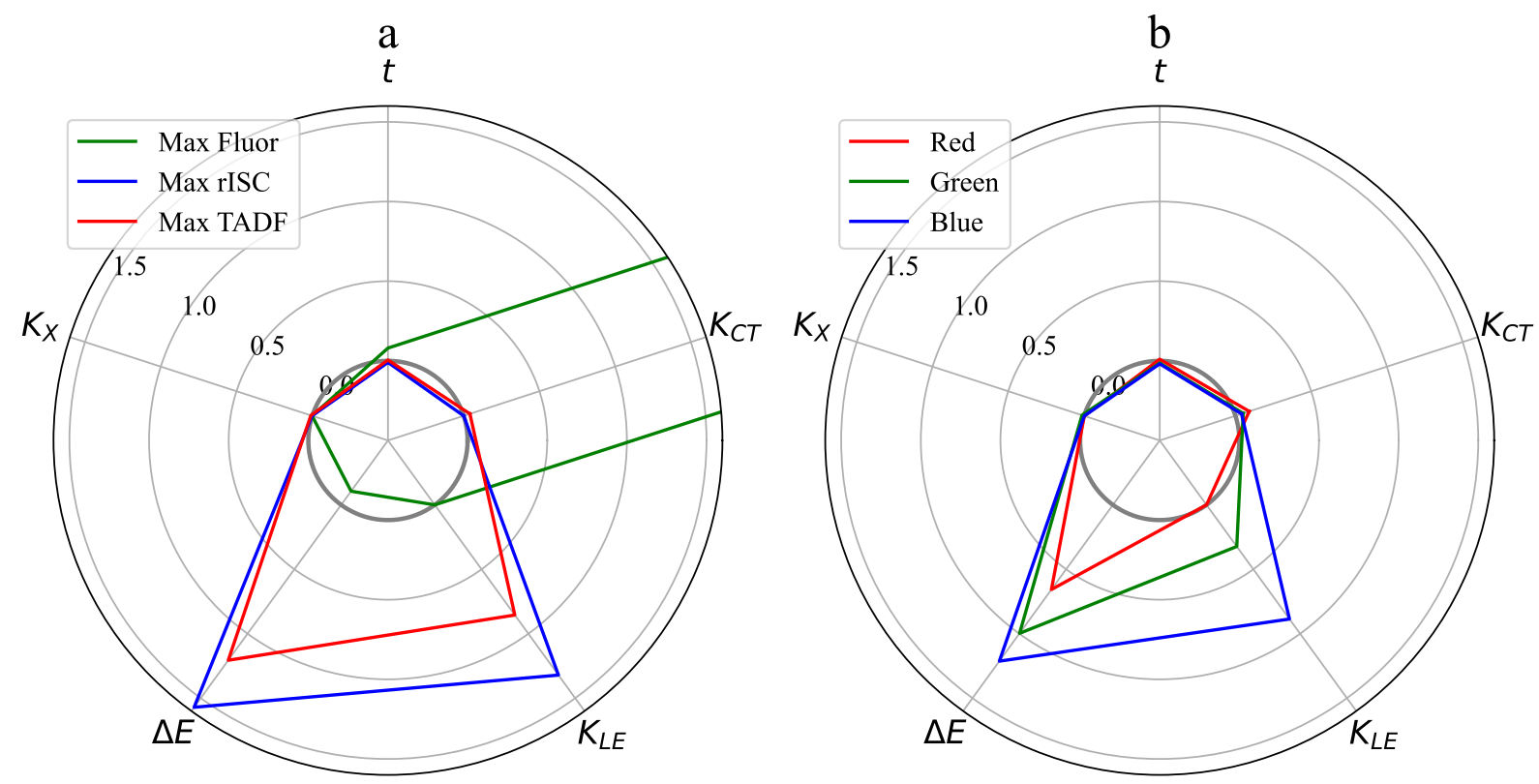

FIG. 5. a) Diabatic Hamiltonians that maximize fluorescence and rISC rates, as well as overall TADF performance. b) Diabatic Hamiltonians that maximize TADF performance constrained by emission color. All values in $\mathrm{eV}$.

increased optical gaps $\left(\Delta E_{\text {opt }}\right)$, but since $t$ does not grow without bounds, we can also infer that excessive values affect oscillator strengths negatively.

More interesting results come from the maximization of $k_{r I S C}$ (blue lines in Figure 5-a). In this case, the best Hamiltonian has $K_{C T}=0 \mathrm{eV}$, which is associated with decreased $\Delta E_{S T}$. In addition, it has $t=-0.01 \mathrm{eV}$, which again suggests some degree of mixture in the diabatic character of the model's adiabatic states. The purpose of this is increasing SOC values, as revealed by the fact that strict SOC optimization is obtained by increasing $t$ indefinitely (Figure SI-4). We also note large values of $\Delta E(1.571 \mathrm{eV})$ and $K_{L E}(1.321 \mathrm{eV})$ in the rISC optimized Hamiltonian, pointing to a requirement that at least one of the adiabatic states with predominant LE character must be of high energy. A similar result is found when optimizing $\Delta E_{S T}$ (Figure SI-4), but not SOC, so we may connect higher $\Delta E$ with lower singlet-triplet gaps. Finally, this particular diabatic Hamiltonian produces as features a $2.253 \mathrm{eV}$ optical gap, but 0 oscillator strength. The intensity of the transition is sacrificed for the sake of a low singlet-triplet gap of $0.028 \mathrm{eV}$ (basically equivalent to thermal energy at room temperature) and a SOC of $0.89 \mathrm{~cm}^{-1}$.

The strict optimization of rISC rates could be relevant for applications such as hyperfluorescence, in which the responsibility for emission is transferred to other molecules ${ }^{53}$. For pure TADF 
applications, simultaneous optimization of fluorescence and rISC rates are required. In this case, the best Hamiltonian found (red lines in Figure 5-a) has $K_{C T}=0.041 \mathrm{eV}$. This slightly elevated value signals a compromise was necessary to improve the oscillator strength. The $t$ coupling in this Hamiltonian was found to be zero. As such, we expect the adiabatic states composing the 4-state model to be nearly pure in character. Similar to the case of pure rISC optimization, the high values of the $K_{L E}$ coupling $(0.855 \mathrm{eV})$ and of $\Delta E(1.206 \mathrm{eV})$ allow us to infer the presence of a high energy state in the model that must correspond to an LE state. Such combination of Hamiltonian elements results in an optical gap of $2.757 \mathrm{eV}$, in the blue region of the spectrum, and this transition becomes allowed with an oscillator strength of 0.012 , which is far from being particularly efficient. The singlet-triplet gap doubles with respect to the pure rISC optimization case to $0.054 \mathrm{eV}$. This value is closer to $K_{C T}$, so considering the options in Equation 9, it becomes clear that we are closer to the first case, which tells us that both $S_{1}$ and $T_{1}$ states in this 4-state model have CT character. The remaining states have LE character, and considering the high $K_{L E}$ coupling, we expect a large energy difference between the two remaining singlet and triplet states. Finally, a SOC of $0.672 \mathrm{~cm}^{-1}$ is produced, lower than when only rISC maximization was the aim.

Finally, we repeat the optimization procedure for TADF performance but now we also enforce a constraint on the optical gap so as to investigate changes in optimization strategy for different OLED colors. Figure 5-b shows optimal diabatic Hamiltonians for $\Delta E_{o p t}$ in the $1.65-2.19 \mathrm{eV}$ (red), 2.19-2.56 eV (green) and 2.56-2.75 eV (blue) range. It can be seen that the three Hamiltonians are similar in shape, but a progressive decrease in $\Delta E$ (from $1.213 \mathrm{eV}$ to $0.657 \mathrm{eV}$ ) and $K_{L E}$ (from $0.886 \mathrm{eV}$ to $0.000 \mathrm{eV}$ ) can be observed when going from blue to red emission. Conversely, an increase in $K_{C T}$ from $0.040 \mathrm{eV}$ to $0.091 \mathrm{eV}$ also takes place. These changes point to a continuous move towards making CT and LE states more similar. I addition, with the energy constraints in place, the optimal value for $t$ drift away from zero $(-0.019 \mathrm{eV},-0.014 \mathrm{eV}$ and $0.010 \mathrm{eV}$ for blue, green and red, respectively). This result points to the necessity of increasing CT/LE mixing for maximum TADF performance. It is also worth mentioning that as one moves from blue to red, the objective function that serves as proxy for TADF performance decreases as well. This indicates that TADF performance is expected to be lower the more redshifted the emission energies are.

Overall, these results clearly indicate the necessity of tuning CT/LE admixture in $S_{1}$ and $T_{1}$ states to allow for the TADF mechanism to work efficiently, highlight the role of vibrations and confirm the 4-state model as an adequate framework for modeling such systems. 


\section{CONCLUSIONS}

In summary, we have presented a diabatization procedure that generates $N$-state models from $N / 2$ singlet and $N / 2$ triplet adiabatic states. The algorithm finds the minimal set of adiabatic states that is able to be well described by CT and LE diabats. This is done for each molecule by attempting to maximize the norm of the adiabatic states that is able to be reproduced by the diabats considered. The procedure was applied to several TADF molecules using as input optimized geometries at different electronic states.

Focusing on the cases that could be well described by a 4-state model, we have been able to provide physical meaning to the different elements of the diabatic Hamiltonian and showed how knowledge of these elements allows for inference on several properties of the model's underlying states. Furthermore, we showed that applying the diabatization procedure to equilibrium geometries of excited states affects mainly the energy difference between CT and LE states and may also modify the amount of CT/LE mixture in the adiabatic states, but does not affect the remaining couplings.

Using a sampling procedure to generate molecular conformations from vibrational motion showed that vibrations are capable of changing importantly the diabatic decomposition of the model states, including changing the nature of the LE state from localized in the acceptor to the donor. Such changes affect all the Hamiltonian elements and consequently all the relevant electronic properties.

With the data from the vibrational analysis we used a simple LASSO model to extract the connections between the Hamiltonian and the electronic properties. This allowed us to determine optimization strategies for maximizing rISC, fluorescence and overall TADF performance. Results showed that optimization of rISC differs from overall TADF optimization by the fact that the latter requires non-zero $K_{C T}$ and $t$ to prevent zero oscillator strengths. This means that there is an optimum amount of mixture between CT and LE diabats in the $S_{1}$ and $T_{1}$ states that is able to achieve the balance between low singlet-triplet gaps and non-zero fluorescence efficiency.

\section{ACKNOWLEDGMENTS}

The authors acknowledge support by a research grant (00028053) from VILLUM FONDEN. 


\section{REFERENCES}

${ }^{1}$ F. B. Dias, K. N. Bourdakos, V. Jankus, K. C. Moss, K. T. Kamtekar, V. Bhalla, J. Santos, M. R. Bryce, and A. P. Monkman, "Triplet harvesting with $100 \%$ efficiency by way of thermally activated delayed fluorescence in charge transfer oled emitters," Advanced Materials 25, 37073714 (2013).

${ }^{2}$ Y. Tao, K. Yuan, T. Chen, P. Xu, H. Li, R. Chen, C. Zheng, L. Zhang, and W. Huang, "Thermally activated delayed fluorescence materials towards the breakthrough of organoelectronics," Advanced materials 26, 7931-7958 (2014).

${ }^{3}$ H. Wang, L. Xie, Q. Peng, L. Meng, Y. Wang, Y. Yi, and P. Wang, "Novel thermally activated delayed fluorescence materials-thioxanthone derivatives and their applications for highly efficient oleds," Advanced Materials 26, 5198-5204 (2014).

${ }^{4}$ T.-A. Lin, T. Chatterjee, W.-L. Tsai, W.-K. Lee, M.-J. Wu, M. Jiao, K.-C. Pan, C.-L. Yi, C.-L. Chung, K.-T. Wong, et al., "Sky-blue organic light emitting diode with 37\% external quantum efficiency using thermally activated delayed fluorescence from spiroacridine-triazine hybrid," Advanced Materials 28, 6976-6983 (2016).

${ }^{5}$ M. Pope, H. Kallmann, and P. Magnante, "Electroluminescence in organic crystals,” The Journal of Chemical Physics 38, 2042-2043 (1963).

${ }^{6} \mathrm{P}$. de Silva, "Inverted singlet-triplet gaps and their relevance to thermally activated delayed fluorescence," The journal of physical chemistry letters 10, 5674-5679 (2019).

${ }^{7}$ J. Ehrmaier, E. J. Rabe, S. R. Pristash, K. L. Corp, C. W. Schlenker, A. L. Sobolewski, and W. Domcke, "Singlet-triplet inversion in heptazine and in polymeric carbon nitrides," The Journal of Physical Chemistry A 123, 8099-8108 (2019).

${ }^{8}$ H. Uoyama, K. Goushi, K. Shizu, H. Nomura, and C. Adachi, "Highly efficient organic lightemitting diodes from delayed fluorescence," Nature 492, 234-238 (2012).

${ }^{9}$ H. Tanaka, K. Shizu, H. Miyazaki, and C. Adachi, "Efficient green thermally activated delayed fluorescence (tadf) from a phenoxazine-triphenyltriazine (pxz-trz) derivative," Chemical Communications 48, 11392-11394 (2012).

${ }^{10}$ K. Goushi, K. Yoshida, K. Sato, and C. Adachi, “Organic light-emitting diodes employing efficient reverse intersystem crossing for triplet-to-singlet state conversion," Nature Photonics 6, 253-258 (2012). 
${ }^{11}$ H. Sun, Z. Hu, C. Zhong, X. Chen, Z. Sun, and J.-L. Brédas, "Impact of dielectric constant on the singlet-triplet gap in thermally activated delayed fluorescence materials," The journal of physical chemistry letters 8, 2393-2398 (2017).

${ }^{12}$ K. Lee and D. Kim, "Local-excitation versus charge-transfer characters in the triplet state: theoretical insight into the singlet-triplet energy differences of carbazolyl-phthalonitrile-based thermally activated delayed fluorescence materials," The Journal of Physical Chemistry C 120, 28330-28336 (2016).

${ }^{13}$ P. K. Samanta, D. Kim, V. Coropceanu, and J.-L. Bredas, "Up-conversion intersystem crossing rates in organic emitters for thermally activated delayed fluorescence: impact of the nature of singlet vs triplet excited states," Journal of the American Chemical Society 139, 4042-4051 (2017).

${ }^{14} \mathrm{D}$. Kim, "A theoretical understanding of the energy difference between singlet and triplet states of oligoacene molecules," International Journal of Quantum Chemistry 116, 651-655 (2016).

${ }^{15}$ D. Kim, "Effects of intermolecular interactions on the singlet-triplet energy difference: a theoretical study of the formation of excimers in acene molecules," The Journal of Physical Chemistry C 119, 12690-12697 (2015).

${ }^{16}$ Y. Gao, T. Su, L. Zhao, Y. Geng, Y. Wu, M. Zhang, and Z.-M. Su, "How does a little difference in structure determine whether molecules have thermally activated delayed fluorescence characteristic or not?" Organic Electronics 50, 70-76 (2017).

${ }^{17}$ Y.-C. Duan, L.-L. Wen, Y. Gao, Y. Wu, L. Zhao, Y. Geng, G.-G. Shan, M. Zhang, and Z.M. Su, "Fluorescence, phosphorescence, or delayed fluorescence?-a theoretical exploration on the reason why a series of similar organic molecules exhibit different luminescence types," The Journal of Physical Chemistry C 122, 23091-23101 (2018).

${ }^{18}$ F. B. Dias, J. Santos, D. R. Graves, P. Data, R. S. Nobuyasu, M. A. Fox, A. S. Batsanov, T. Palmeira, M. N. Berberan-Santos, M. R. Bryce, et al., "The role of local triplet excited states and $\mathrm{d}$-a relative orientation in thermally activated delayed fluorescence: photophysics and devices," Advanced Science 3, 1600080 (2016).

${ }^{19}$ T. Hosokai, H. Noda, H. Nakanotani, T. Nawata, Y. Nakayama, H. Matsuzaki, and C. Adachi, "Solvent-dependent investigation of carbazole benzonitrile derivatives: does the 3 le- 1 ct energy gap facilitate thermally activated delayed fluorescence?” Journal of Photonics for Energy 8, $032102(2018)$. 
${ }^{20}$ L. Gan, K. Gao, X. Cai, D. Chen, and S.-J. Su, “Achieving efficient triplet exciton utilization with large $\delta$ e st and nonobvious delayed fluorescence by adjusting excited state energy levels," The journal of physical chemistry letters 9, 4725-4731 (2018).

${ }^{21}$ J. Gibson, A. P. Monkman, and T. J. Penfold, "The importance of vibronic coupling for efficient reverse intersystem crossing in thermally activated delayed fluorescence molecules," ChemPhysChem 17, 2956 (2016).

${ }^{22}$ T. Penfold, F. Dias, and A. P. Monkman, "The theory of thermally activated delayed fluorescence for organic light emitting diodes," Chemical communications 54, 3926-3935 (2018).

${ }^{23}$ T. Van Voorhis, T. Kowalczyk, B. Kaduk, L.-P. Wang, C.-L. Cheng, and Q. Wu, "The diabatic picture of electron transfer, reaction barriers, and molecular dynamics," Annual review of physical chemistry 61, 149-170 (2010).

${ }^{24}$ H. Köppel, W. Domcke, and L. S. Cederbaum, "Multimode molecular dynamics beyond the born-oppenheimer approximation,” Advances in chemical physics 57, 59-246 (1984).

${ }^{25}$ U. Manthe and H. Köppel, "Dynamics on potential energy surfaces with a conical intersection: Adiabatic, intermediate, and diabatic behavior,” The Journal of chemical physics 93, 1658-1669 (1990).

${ }^{26}$ F. Gadea and M. Pelissier, "Approximately diabatic states: A relation between effective hamiltonian techniques and explicit cancellation of the derivative coupling," The Journal of chemical physics 93, 545-551 (1990).

${ }^{27}$ P. de Silva, C. A. Kim, T. Zhu, and T. Van Voorhis, "Extracting design principles for efficient thermally activated delayed fluorescence (tadf) from a simple four-state model," Chemistry of Materials 31, 6995-7006 (2019).

${ }^{28}$ P. Ramos and M. Pavanello, "Low-lying excited states by constrained dft," The Journal of chemical physics 148, 144103 (2018).

${ }^{29} \mathrm{Q}$. Wu and T. Van Voorhis, "Constrained density functional theory and its application in longrange electron transfer," Journal of Chemical Theory and Computation 2, 765-774 (2006).

${ }^{30} \mathrm{Q}$. Wu and T. Van Voorhis, "Extracting electron transfer coupling elements from constrained density functional theory," The Journal of chemical physics 125, 164105 (2006).

${ }^{31}$ S. Difley and T. Van Voorhis, "Exciton/charge-transfer electronic couplings in organic semiconductors," Journal of chemical theory and computation 7, 594-601 (2011).

${ }^{32}$ J. Liu, Y. Zhang, P. Bao, and Y. Yi, "Evaluating electronic couplings for excited state charge transfer based on maximum occupation method $\delta$ scf quasi-adiabatic states," Journal of chemical 
theory and computation 13, 843-851 (2017).

${ }^{33}$ T. Kowalczyk, S. R. Yost, and T. V. Voorhis, "Assessment of the $\delta$ scf density functional theory approach for electronic excitations in organic dyes," The Journal of chemical physics 134, $054128(2011)$.

${ }^{34}$ W. Liu, B. Lunkenheimer, V. Settels, B. Engels, R. F. Fink, and A. Köhn, “A general ansatz for constructing quasi-diabatic states in electronically excited aggregated systems," The Journal of chemical physics 143, 084106 (2015).

${ }^{35}$ W. Liu, S. Canola, A. Köhn, B. Engels, F. Negri, and R. F. Fink, “A model hamiltonian tuned toward high level ab initio calculations to describe the character of excitonic states in perylenebisimide aggregates," Journal of computational chemistry 39, 1979-1989 (2018).

${ }^{36}$ Y. Xie, S. Jiang, J. Zheng, and Z. Lan, "Construction of vibronic diabatic hamiltonian for excited-state electron and energy transfer processes," The Journal of Physical Chemistry A 121, 9567-9578 (2017).

${ }^{37}$ A. E. Reed and F. Weinhold, "Natural localized molecular orbitals," The Journal of chemical physics 83, 1736-1740 (1985).

${ }^{38}$ H. Nakamura and D. G. Truhlar, "The direct calculation of diabatic states based on configurational uniformity," The Journal of Chemical Physics 115, 10353-10372 (2001).

${ }^{39}$ J.-D. Chai and M. Head-Gordon, "Long-range corrected hybrid density functionals with damped atom-atom dispersion corrections," Physical Chemistry Chemical Physics 10, 6615-6620 (2008).

${ }^{40}$ M. J. Frisch, G. W. Trucks, H. B. Schlegel, G. E. Scuseria, M. A. Robb, J. R. Cheeseman, G. Scalmani, V. Barone, G. A. Petersson, H. Nakatsuji, X. Li, M. Caricato, A. V. Marenich, J. Bloino, B. G. Janesko, R. Gomperts, B. Mennucci, H. P. Hratchian, J. V. Ortiz, A. F. Izmaylov, J. L. Sonnenberg, D. Williams-Young, F. Ding, F. Lipparini, F. Egidi, J. Goings, B. Peng, A. Petrone, T. Henderson, D. Ranasinghe, V. G. Zakrzewski, J. Gao, N. Rega, G. Zheng, W. Liang, M. Hada, M. Ehara, K. Toyota, R. Fukuda, J. Hasegawa, M. Ishida, T. Nakajima, Y. Honda, O. Kitao, H. Nakai, T. Vreven, K. Throssell, J. A. Montgomery, Jr., J. E. Peralta, F. Ogliaro, M. J. Bearpark, J. J. Heyd, E. N. Brothers, K. N. Kudin, V. N. Staroverov, T. A. Keith, R. Kobayashi, J. Normand, K. Raghavachari, A. P. Rendell, J. C. Burant, S. S. Iyengar, J. Tomasi, M. Cossi, J. M. Millam, M. Klene, C. Adamo, R. Cammi, J. W. Ochterski, R. L. Martin, K. Morokuma, O. Farkas, J. B. Foresman, and D. J. Fox, “Gaussian 16 Revision C.01,” (2016), gaussian Inc. Wallingford CT. 
${ }^{41}$ Y. Shao, Z. Gan, E. Epifanovsky, A. T. Gilbert, M. Wormit, J. Kussmann, A. W. Lange, A. Behn, J. Deng, X. Feng, et al., "Advances in molecular quantum chemistry contained in the q-chem 4 program package,” Molecular Physics 113, 184-215 (2015).

${ }^{42}$ E. Glendening, A. Reed, J. Carpenter, F. Weinhold, et al., "Nbo version 3.1,” (1998).

${ }^{43}$ J. Lee, N. Aizawa, M. Numata, C. Adachi, and T. Yasuda, "Versatile molecular functionalization for inhibiting concentration quenching of thermally activated delayed fluorescence," Advanced Materials 29, 1604856 (2017).

${ }^{44}$ G. Grybauskaite-Kaminskiene, D. Volyniuk, V. Mimaite, O. Bezvikonnyi, A. Bucinskas, G. Bagdziunas, and J. V. Grazulevicius, "Aggregation-enhanced emission and thermally activated delayed fluorescence of derivatives of 9-phenyl-9h-carbazole: effects of methoxy and tert-butyl substituents," Chemistry-A European Journal 24, 9581-9591 (2018).

${ }^{45}$ R. S. Nobuyasu, Z. Ren, G. C. Griffiths, A. S. Batsanov, P. Data, S. Yan, A. P. Monkman, M. R. Bryce, and F. B. Dias, "Rational design of tadf polymers using a donor-acceptor monomer with enhanced tadf efficiency induced by the energy alignment of charge transfer and local triplet excited states," Advanced Optical Materials 4, 597-607 (2016).

${ }^{46}$ N. Aizawa, C.-J. Tsou, I. S. Park, and T. Yasuda, “Aggregation-induced delayed fluorescence from phenothiazine-containing donor-acceptor molecules for high-efficiency non-doped organic light-emitting diodes," Polymer Journal 49, 197-202 (2017).

${ }^{47}$ J. Lee, N. Aizawa, and T. Yasuda, "Isobenzofuranone-and chromone-based blue delayed fluorescence emitters with low efficiency roll-off in organic light-emitting diodes," Chemistry of Materials 29, 8012-8020 (2017).

${ }^{48}$ K. Nasu, T. Nakagawa, H. Nomura, C.-J. Lin, C.-H. Cheng, M.-R. Tseng, T. Yasuda, and C. Adachi, "A highly luminescent spiro-anthracenone-based organic light-emitting diode exhibiting thermally activated delayed fluorescence," Chemical Communications 49, 10385-10387 (2013).

${ }^{49}$ T. J. Penfold, E. Gindensperger, C. Daniel, and C. M. Marian, "Spin-vibronic mechanism for intersystem crossing," Chemical reviews 118, 6975-7025 (2018).

${ }^{50}$ L. E. de Sousa and P. de Silva, "Unified framework for photophysical rate calculations in tadf molecules," Journal of Chemical Theory and Computation 17, 5816-5824 (2021).

${ }^{51}$ R. Tibshirani, "Regression shrinkage and selection via the lasso," Journal of the Royal Statistical Society: Series B (Methodological) 58, 267-288 (1996). 
${ }^{52}$ F. Pedregosa, G. Varoquaux, A. Gramfort, V. Michel, B. Thirion, O. Grisel, M. Blondel, P. Prettenhofer, R. Weiss, V. Dubourg, J. Vanderplas, A. Passos, D. Cournapeau, M. Brucher, M. Perrot, and E. Duchesnay, “Scikit-learn: Machine learning in Python,” Journal of Machine Learning Research 12, 2825-2830 (2011).

${ }^{53}$ L. E. de Sousa, L. dos Santos Born, P. H. de Oliveira Neto, and P. de Silva, "Triplet-to-singlet exciton transfer in hyperfluorescent oled materials," (2021). 